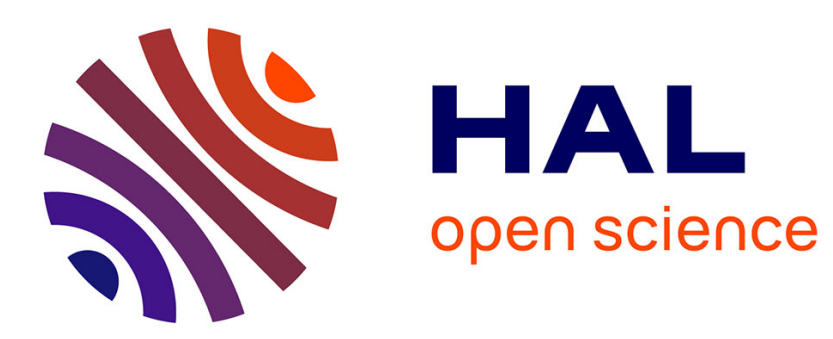

\title{
Pilot-based parametric channel estimation algorithm for DCO-OFDM-based visual light communications
}

\author{
Xuewen Qian, Honggui Deng, Hailang He
}

\section{To cite this version:}

Xuewen Qian, Honggui Deng, Hailang He. Pilot-based parametric channel estimation algorithm for DCO-OFDM-based visual light communications. Optics Communications, 2017, 400, pp.150-155. 10.1016/j.optcom.2017.05.020 . hal-01923662

\section{HAL Id: hal-01923662 \\ https://hal.science/hal-01923662}

Submitted on 24 Nov 2020

HAL is a multi-disciplinary open access archive for the deposit and dissemination of scientific research documents, whether they are published or not. The documents may come from teaching and research institutions in France or abroad, or from public or private research centers.
L'archive ouverte pluridisciplinaire HAL, est destinée au dépôt et à la diffusion de documents scientifiques de niveau recherche, publiés ou non, émanant des établissements d'enseignement et de recherche français ou étrangers, des laboratoires publics ou privés. 


\title{
Pilot-based Parametric Channel Estimation algorithm for DCO-OFDM-based Visual Light Communications
}

\author{
Xuewen Qian ${ }^{\mathrm{a}}$, Honggui Deng ${ }^{\mathrm{a}}$, Hailang $\mathrm{He}^{\mathrm{b}}$ \\ ${ }^{a}$ Dept. of Physics and Electronics, Central South University, Changsha, Hunan, China. \\ ${ }^{b}$ Dept. of Information Engineering, Shaoyang University, Shaoyang, Hunan, China.
}

\begin{abstract}
Due to wide modulation bandwidth in optical communication, multipath channels may be non-sparse and deteriorate communication performance heavily. Traditional compressive sensing-based channel estimation algorithm cannot be employed in this kind of situation. In this paper, we propose a practical parametric channel estimation algorithm for orthogonal frequency division multiplexing (OFDM)-based visual light communication (VLC) systems based on modified zero correlation code (ZCC) pair that has the impulse-like correlation property. Simulation results show that the proposed algorithm achieves better performances than existing least squares (LS)-based algorith$\mathrm{m}$ in both bit error ration (BER) and frequency response estimation.
\end{abstract}

Keywords:

OFDM, VLC, zero correlation code pair, LS, channel estimation.

\section{Introduction}

Visual light communication (VLC) technology [1] is becoming increasingly important for future communication systems due to its ability to provide data transmissions and illumination with high security and energy conservation. Besides, the light emitting diode (LED) as one of the most popular and important light sources can promote the widespread use of VLC systems. However, multi-path distortion caused by the reflections can heavily reduce the communication qualities of VLC systems. Thus, orthogonal frequency division multiplexing (OFDM) technique is introduced into VLC systems to combat the multi-path effect. Due to intensity modulation and direct detection in most VLC systems, many OFDM-based VLC systems $[1,2,3,4,5,6]$ 
resort to the direct current-biased OFDM modulation schemes to enable signals to be positive which is different from radio OFDM systems.

To ensure stable and efficient transmissions of OFDM-based VLC systems, channel state information (CSI) must be estimated. One algorithm is exploiting the pilots which can be generated by block-type and scattered schemes $[1,3,4]$. Another algorithm is based on the preamble which is a kind of block-type pilot symbol. Mostly, OFDM-based VLC systems use the least squares (LS) algorithms [7] to acquire the channel frequency response. This algorithm is rather simple and easy to implement, but is sensitive to noise. The linear minimum mean square error (LMMSE) algorithm which is a modified algorithm of minimum mean square error (MMSE) [8, 9] achieves better performances than LS algorithm. However, the calculation complexity is still extremely large and has to know the channel statical information in advance which makes the two kinds of algorithms less pragmatic to be employed.

Bajwa et al [10] have introduced compressed sensing technique into channel estimation and applied it in double selective channel situations for radio communications. It firstly estimates the number of paths, and then acquires the delay profiles using estimation of signal parameters via rotational invariance techniques (ESPRIT) [11]. The two processes require an extremely large amount of calculations. Finally, it calculates the gain of each path by solving an optimal problem. It is also called parametric channel estimation algorithm. This technique can improve estimation performances and decrease the need for pilots which contributes to a higher spectrum efficiency. This kind of algorithm is based on the assumption that the communication channel is sparse in discrete time domain $[12,13]$. In VLC application scenarios, sparse channel does exist in some situations such as the outdoor car-to-car communication using the headlamps to transmit signals. It is evaluated by SeokJu Lee et. al [5]. If the channel is not sparse, compressed sensing technique does not work well. It is pointed in the paper of Xudong Zhu et. al [13] in which they proposed a sparsity-aware channel estimation algorithm for radio communication systems. In indoors and near field communication situations, the maximum path delay of multipath channel may exceed 60ns[14]. And the modulation bandwidth may exceed $500 \mathrm{MHz} / \mathrm{s}$ with the help of $\mu$-LED or laser diode[15]. In this case, the channel is no longer sparse [16] for these systems. Thus, compressed sensing-based channel estimation algorithm is useless.

In radio communication systems, some compressed sensing-based algo- 
rithms coarsely estimate the gains and delays of channels by using the constant amplitude zero autocorrelation code (CAZAC) [17]. However, CAZAC sequences cannot be used in VLC systems due to the intensity modulation and direct detection (DD) as the amplitude of CAZAC is constant. Also, all the subcarriers must be exploited to generate CAZAC sequences which means this sequence cannot be used in pilot-based channel estimation algorithms. Motivated by the parametric channel estimation algorithm and CAZAC sequences, we propose a novel algorithm based on the modified zero correlation code (ZCC) pair to acquire CSI. Compared with CAZAC sequences, the modified ZCC pair can be generated into real value signals by using pilot data. Thus, modified ZCC pair can be used in VLC systems. The rest is organized as follows. In Section II, the system model is presented, including the channel model and DCO-OFDM-based VLC system. Then, we give detailed descriptions on the modified ZCC pair and our algorithm in Section III. In Section IV, performance and simulation results are analysed. In the end, the conclusion is drawn in Section V.

\section{System Model}

\subsection{DCO-OFDM-based VLC system}

In order to make the transmitted signal be positive, a common scheme is to add a direct-current (DC) offset value to signals. In this case, this kind of OFDM system is called DC offset OFDM (DCO-OFDM). At the receiver, we just need to subtract a DC value and the signal we get is what we need. So we do not need to consider this process.

To overcome the effect of multipath, the cyclic prefix $(\mathrm{CP})$ is introduced in the OFDM systems. Considering a DCO-OFDM system with $N$ sub-carriers, the baseband model is given as

$$
x(n)=\frac{1}{\sqrt{N}} \sum_{i=0}^{N-1} X_{i} e^{j 2 \pi \frac{n i}{N}},-N_{C P} \leq n \leq N-1
$$

where $n$ denotes the index of signal in time domain and $X_{i}$ denotes the modulated data on the k-th sub-carrier. The $N_{C P}$ represents CP length. To make $x(n)$ be real, $X_{i}$ must follow the Hermite symmetry property which is shown as

$$
X=\left[X_{0}, X_{1}, \ldots, X_{N / 2-1}, X(N / 2), X_{N / 2-1}^{*}, \ldots, X_{1}^{*}\right]
$$


Besides, $X_{0}$ and $X(N / 2)$ should be real.

The whole system is shown in Fig.1 and the mapping type is multilevel quadrature amplitude modulation (MQAM). Then the mapped data are inverse Fourier transformed into real-valued data and $\mathrm{CP}$ is added. After that, the sequence will be transmitted through digital to analog converter (DAC) and LED transmitter. We add a DC bias voltage for the LED transmitter because signals of the VLC systems are non-negative. After signal coming through line of sight (LoS) or non-line of sight (NLoS) path channel, the photodetectors (PD) receive signals by direct detection. Then the $\mathrm{CP}$ is removed and received data are Fourier transformed, demodulated and recovered into the original data.

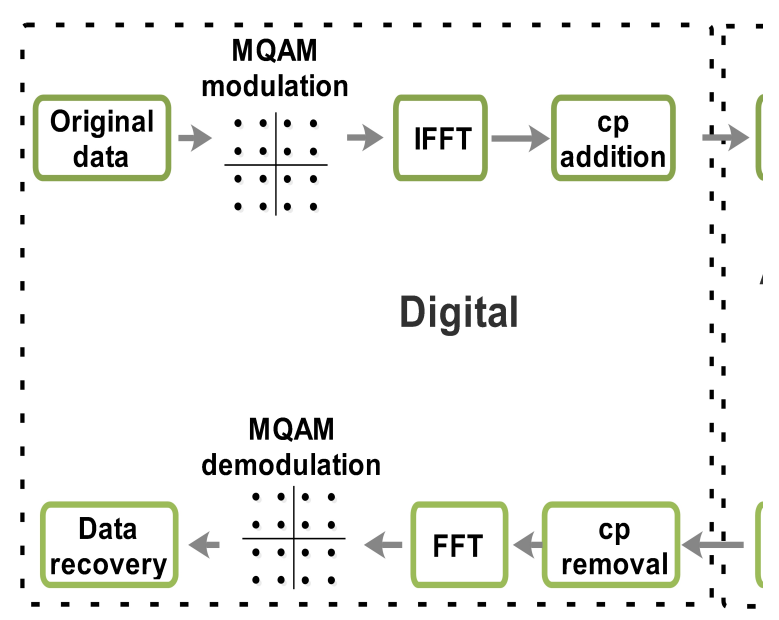

Figure 1: Indoor geometric distribution model of the system and the schematic of a DCOOFDM-based VLC system

The effect of multipath channel can be written as

$$
y(n)=\sum_{l=0}^{L-1} h(l) x\left(n-\tau_{l}\right)+w(n)
$$

where $h(l)$ represents the VLC channel time domain response and $w(n)$ denotes the total noise consist of the ambient light shot noise and thermal noise which can be modeled as white Gaussian noise[14].

The received signal in frequency domain can be expressed as

$$
Y(k)=X(k) H(k)+W(k),
$$


where $H(k)$ follows the equation as

$$
H(k)=\sum_{l=0}^{L-1} g(l) e^{-j 2 \pi \frac{\tau_{l} k}{N}}, 0 \leq k \leq N-1
$$

As long as we acquire the gains and delays of multipath channels, we would get the channel impulse response. Thus, the main task is estimating the gains and delays of multipath channels.

\subsection{VLC Channel Model}

A realistic VLC channel is determined by many factors, such as realistic light source, different types of reflections and the light wavelength. For example, in home environment, the LOS response depends on the LOS length and its power is bigger than that of NLOS components caused by a large number of reflections from walls, floor and other objects like glass shown in Fig. 2 .

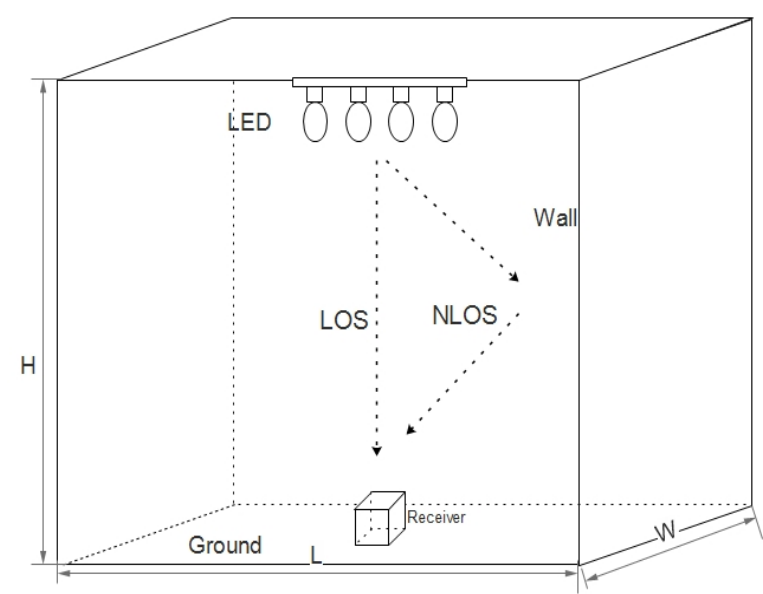

Figure 2: Indoor geometric distribution model of the system

As pointed in [18], the maximum path delay in home environment is about $60 \mathrm{~ns}$ and there is often one LOS response while the NLOS responses are extremely small. However in manufacturing cell environment, the maximum path delay may exceed 80ns. Also, there are more strong LOS responses as many light traces are reflected by metal materials and NLOS responses cannot be neglected. 
Overall, we can model the VLC channel as multipath channel. The VLC channel can be modeled as

$$
h(\tau)=\sum_{l=0}^{L-1} g(l) \delta\left(\tau-\tau_{l}\right),
$$

where $g(l)$ and $\tau_{l}$ denote the gain and delay of the l-th transmission path, $\delta(\tau)$ denotes the Dirac function and $L$ represents the number of path. $h(\tau)$ can be made discrete at a sampling speed. For simplicity, we assume that $\tau_{l}$ is an integer. Since VLC systems can only transfer intensity signals, $g(l) \geq 0$.

\section{Modified Zero-correlation-code pair and proposed algorithm}

\subsection{Modified Zero-correlation-code pair}

In previous work, Zero-correlation-code (ZCC) pair [6] is proposed to synchronize signal in OFDM-based VLC systems by using the impulse-like periodic correlation relationship. This can be exploited in pilot-based channel estimation with some modifications.

Let $a_{p}$ and $b_{p+q}$ be the results that $A_{n}$ and $B_{n}$ are inverse Fourier transformed into with different order. Then, the two parts can be expressed as

$$
\begin{gathered}
a_{p}=\frac{1}{\sqrt{N}} \sum_{n=0}^{N-1} A_{n} \cdot e^{j 2 \pi p n / N}, \\
b_{p+q}=\frac{1}{\sqrt{N}} \sum_{m=0}^{N-1} B_{m} \cdot e^{j 2 \pi(p+q) m / N} .
\end{gathered}
$$

If $A_{n} * B_{n}^{*}=1$, there will be periodic correlation relationship as

$$
\begin{aligned}
& \sum_{p=0}^{N-1} a_{p} \cdot b_{p+q}^{*} \\
= & \frac{1}{N} \sum_{p=0}^{N-1} \sum_{n=0}^{N-1} \sum_{m=0}^{N-1} A_{n} \cdot e^{j 2 \pi p n / N} \cdot B_{m}^{*} e^{-j 2 \pi(p+q) m / N} \\
= & \frac{1}{N} \sum_{p=0}^{N-1} \sum_{n=0}^{N-1} \sum_{m=0}^{N-1} A_{n} \cdot B_{m}^{*} \cdot e^{j 2 \pi p(n-m) / N} \cdot e^{-j 2 \pi q m / N}
\end{aligned}
$$




$$
\begin{aligned}
& =\sum_{n=0}^{N-1} \sum_{m=0}^{N-1} A_{n} \cdot B_{m}^{*} \cdot \delta(n-m) \cdot e^{-j 2 \pi q m / N} \\
& =\sum_{n=0}^{N-1} A_{n} \cdot B_{n}^{*} \cdot e^{-j 2 \pi q n / N} \\
& =\delta(q)
\end{aligned}
$$

where $\delta(q)$ denotes the Dirac sequence and $\delta(0)=1$ and $\delta(N)=1$.

In channel estimation, scattered pilots are mostly wide used. Inspired by Eq. (9), we just exploit the scattered sub-carriers with a fixed step $P$ which means that

$$
A_{n} \cdot B_{n}^{*}=1, n=i+k \cdot P,
$$

Eq. (9) can be derived as

$$
\begin{aligned}
\sum_{p=0}^{N-1} a_{p} b_{p+q}^{*} & =\sum_{n=0}^{N-1} A_{n} \cdot B_{n}^{*} \cdot e^{-j 2 \pi q n / N} \\
& =e^{-j 2 \pi q i / N} \sum_{n=0}^{\left\lfloor\frac{N}{P}\right\rfloor-1} e^{\frac{-j 2 \pi q P n}{N}} \\
& =e^{\frac{-j 2 \pi q i}{N}} \frac{1-e^{\frac{-j 2 \pi q P\left\lfloor\frac{N}{P}\right\rfloor}{N}}}{1-e^{\frac{-j 2 \pi q P}{N}}},
\end{aligned}
$$

which reflects that the correlation result is approximately impulse-like. If the result of $\frac{N}{P}$ is an integer, Eq.(11) is totally periodically impulse-like.

$$
\sum_{p=0}^{N-1} a_{p} b_{p+q}^{*}=\frac{N}{P} \cdot e^{-j 2 \pi q i / N} \delta(q P),
$$

And the period is $\frac{N}{P}$. This property can be used in channel estimation.

\subsection{Proposed algorithm}

Inspired by some compressed sensing-based channel estimation algorithms in radio communications systems using CAZAC [17], the proposed algorithm's main part is to estimate the channel characteristics via modified ZCC pair. 
If we denote $x_{a}(n)$ as the transmitted pilot data in one OFDM symbol and $x_{b}(n)$ as the pair symbol that can be generated via Eq.(7) and Eq.(8) with the relationship of Eq.(10), there will be $\sum_{n=0}^{N-1} x_{a}(n) x_{b}^{*}(n)=\left\lfloor\frac{N}{P}\right\rfloor$ and $\sum_{n=0}^{N-1}\left(x(n+i)-x_{a}(n+i)\right) x_{b}^{*}(n)=0$ when $-N_{C P} \leq i \leq 0$. The correlation calculation is absolutely periodic within the whole symbol region and aperiodic out of the range shown in Fig. 3. Therefore, the original pilot data

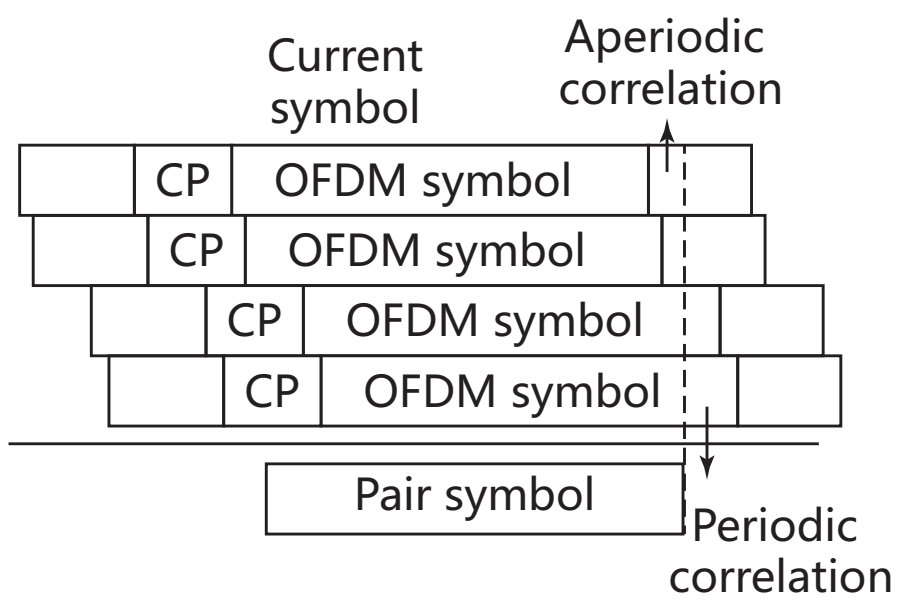

Figure 3: The illustration of multipath effect on the correlation calculation

should be carefully chosen.

Recently, in the fifth generation $(5 \mathrm{G})$ communication systems, the communication systems of Physical layer for dynamic spectrum access and cognitive radio (PHYDYAS) [19] is proposed. The prototype filter they proposed has good performance in many aspects. We introduce this into the modified ZCC pair sequence generation.

The first thing to do is to generate the original unmodulated data $g$.

Step1: Define $S$ as a vector of $N / 8+1$ length consisting of 1 and -1 : $S=[1, \cdots 1, \cdots-1, \ldots-1]^{T}$.

Step2: $S$ is multiplied by a matrix $\operatorname{diag}\left(\left[1, j,-1, j^{3}, \ldots j^{N / 8-1}, j^{N / 8}\right]\right)$ to be $S S$. This is also called offset quadrature amplitude modulation process.

Step3: Define $S S S$ as a vector with Hermite property via $S S S=\left[S S(0), S S(1), \cdots S S(N / 8), S S^{*}(N / 8-1), \cdots S S^{*}(1)\right]^{T}$.

Step4: Finally, we get the original data $g$ before IFFT process using 

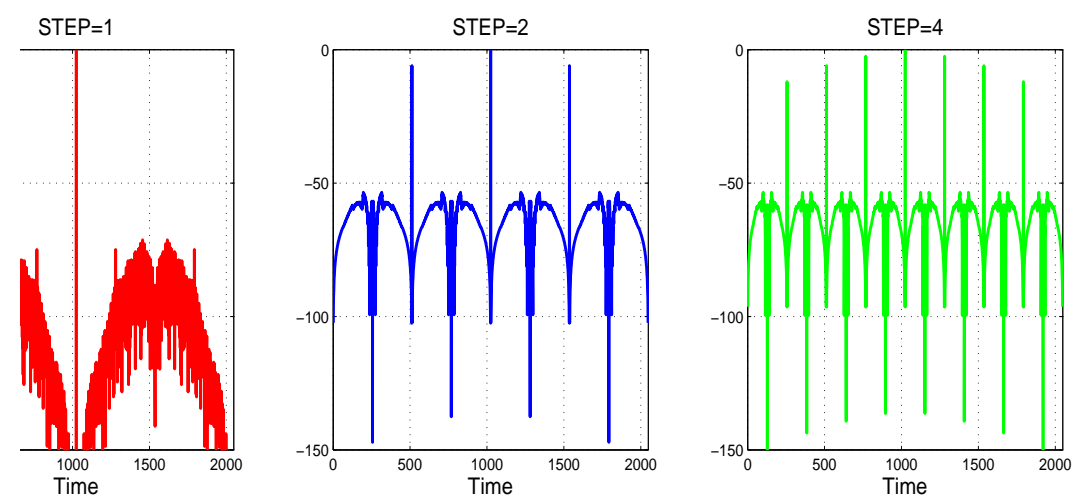

Figure 4: The aperiodic correlation results of modified ZCC pair using PHYDYAS filter with different sub-carrier steps

PHYDYAS filter via $g=G \cdot S S S$. Here, $G$ is expressed as

$$
G=\left(\begin{array}{cccc}
G_{0} & 0 & \ldots & 0 \\
G_{1} & G_{3} & \ldots & 0 \\
G_{2} & G_{2} & \ldots & 0 \\
G_{3} & G_{1} & \ldots & 0 \\
0 & G_{0} & \ldots & 0 \\
\vdots & 0 & \ddots & \vdots \\
G_{3} & 0 & \ldots & G_{1} \\
G_{2} & 0 & \ldots & G_{2} \\
G_{1} & 0 & \ldots & G_{3}
\end{array}\right)_{N \times \frac{N}{4}}
$$

where $G_{0}, G_{1}, G_{2}$ and $G_{3}$ are given in [19].

We then get original pilot data by spreading elements of $g$ to each pilot sub-carriers. For example, if the sub-carrier step is 4, then $X_{0}=g(0)$ and $X_{4}=g(1)$. Also, $X_{N-4}=g^{*}(1)$ and etc. If the sub-carrier step is 2 , then $X_{0}=g(0), X_{2}=g(1)$ and $X_{N-2}=g^{*}(1)$. Then $x_{a}(n)$ and $x_{b}(n)$ can be generated via Eq.(7) and Eq.(8) with the relationship of Eq.(10) and has good aperiodic correlation results shown in Fig.(4).

If we choose all the sub-carriers as pilots, then the nearby symbols will not affect correlation results. In this case, we can get each path gain via the following equation.

$$
\bar{h}(k)=\sum_{n=0}^{N-1} y(n+k) x_{b}^{*}(n)
$$




$$
\begin{gathered}
=\sum_{n=0}^{N-1} \sum_{l=0}^{L-1}(h(l) x(n+k-l)+w(n+k)) x_{b}^{*}(n) \\
=\sum_{l=0}^{L-1} h(l) \sum_{n=0}^{N-1} x(n+k-l) x_{b}^{*}(n)+\tilde{w}(k) \\
\bar{h}(k)=\sum_{l=0}^{L-1} h(l) \delta(k-l)+\tilde{w}(k)
\end{gathered}
$$

When we just choose a small amount of sub-carriers, $\sum_{n=0}^{N-1} x(n+k-$ l) $x_{b}^{*}(n)=\delta(k-l)$ is not equal to $\delta(k-l)$, thus Eq.(15) is no longer equal. It should be multiplied with a matrix given in subsequent discuss. We rewrite Eq.(14) in matrix form as

$$
\bar{h}=A h+w
$$

where $\bar{h}=[\bar{h}(0), \bar{h}(1), \ldots, \bar{h}(L-1)]$ and $h=[h(0), h(1), \ldots, h(L-1)]$. The $\bar{h}$ is the coarse estimated channel response and $h$ is the real channel response. The $A$ can be expressed as

$$
A=\left(\begin{array}{cccc}
A(0) & A(1) & \ldots & A(L-1) \\
A(-1) & A(0) & \ldots & A(L-2) \\
\vdots & & \ddots & \vdots \\
A(-L+1) & A(-L+2) & \ldots & A(0)
\end{array}\right)
$$

where

$$
A(k)=\left\{\begin{array}{cc}
\sum_{n=0}^{N-1} x_{a}(n+k) x_{b}^{*}(n) & k \leq 0 \\
\sum_{n=0}^{N-1-k} x_{a}(n+k) x_{b}^{*}(n) & k \geq 0
\end{array}\right.
$$

Therefore, the gain estimation is calculated via $h=A^{-1} \bar{h}$.

Therefore, the whole algorithm can be listed as:

1. Parameters: IFFT length $N$, pilot step STEP

2. Initialization: Spread elements of $g$ to pilot sub-carriers and generate $x_{a}(n)$ and $x_{b}(n)$. Generate matrix $A$ according to Eq.(17) and Eq.(18).

3. Calculate the correlation results between $y(n)$ and $x_{b}(n)$ as $\bar{h}$.

4. Calculate the estimated channel impulse response $h=A^{-1} \bar{h}$

5. Calculate the channel frequency response via Eq.(5) 


\section{Simulation and Analysis}

We compared the mean squared error (MSE) and bit error rate (BER) performances of proposed algorithm with the LS-based channel estimation algorithm. In this paper, LS-based channel estimation algorithm is based on spline interpolation of scattered pilot.

Table 1: The parameters of simulation

\begin{tabular}{lc}
\hline Parameters & Value \\
\hline Number of subcarriers & 512 \\
CP length & 64 \\
Frame length & 16 OFDM symbols \\
Bandwidth & $500 \mathrm{MHz}$ \\
Constellation order & $16 \mathrm{QAM}$ \\
Pilot distribution & Scattered \\
Simulation trial number & 100000 \\
Room area W $\times \mathrm{H} \times \mathrm{L}$ & $2 \times 4 \times 3 \mathrm{~m}^{3}$ \\
Receiving node $(\mathrm{PD})$ area & $1 \mathrm{~cm}^{2}$ \\
Conversion efficient of photoelectricity & $0.5 \mathrm{~A} / \mathrm{W}$ \\
Receiver field of view $($ FOV $)$ & $60^{\circ}$ \\
\hline
\end{tabular}

The VLC channel we use is the indoor manufacturing cell channel given by [20]. As to the signal-to-noise ratio, in paper [1], authors have tested the OFDM-based VLC systems using common LEDs and revealed that the average SNR values are over 18dB. If we choose better LED lamps, the average SNR value will exceed $20 \mathrm{~dB}$. Therefore we set the SNR range as [20dB 40dB].

LS-based channel estimation algorithm is a kind of frequency-domain channel estimation method. Once we obtain the channel frequency domain response of each pilot subcarrier, we can recover the whole frequency response via spline or linear interpolation. However, if we choose linear interpolation, the systems will suffer from performance degradation. If we choose spline interpolation, the performance will be improved but systems have to resort to complex calculations to fulfill this task. The inverse of diagonally dominant matrix needs to be solved to acquire the parameters of spline interpolation process before we calculate the channel frequency response. Apart from the calculation of inverse matrix, the LS-based channel estimation algorithm requires at least $3 N$ multiplication process to recover the whole CSI. 
The proposed algorithm is a kind of time-domain channel estimation algorithm which is different from LS-based algorithm. Since we do not know the real channel length, we regard the channel length as $N_{C P}$ for all kinds of channels assuming that the channel length is smaller than CP length. We can directly store the inverse matrix of $A$ to reduce computational complexity. If $N_{C P}<\frac{N}{S T E P}$, the diagonal values of $A$ are roughly near to 1 and the rest are rather small which means $A$ turns into an identity matrix. Thus, our algorithm is less computationally complex compared with LS-based algorithm. As to the channel recovery, what we need to do is to calculate the channel frequency response via Eq.(5) which means we can input the gain and delay data into the FFT module. In this case, we do not need extra computation and the whole computation complexity of our algorithm is $N_{C P} \cdot\left(N+N_{C P}\right)$. Overall, our algorithm is less complex than LS-based algorithm.

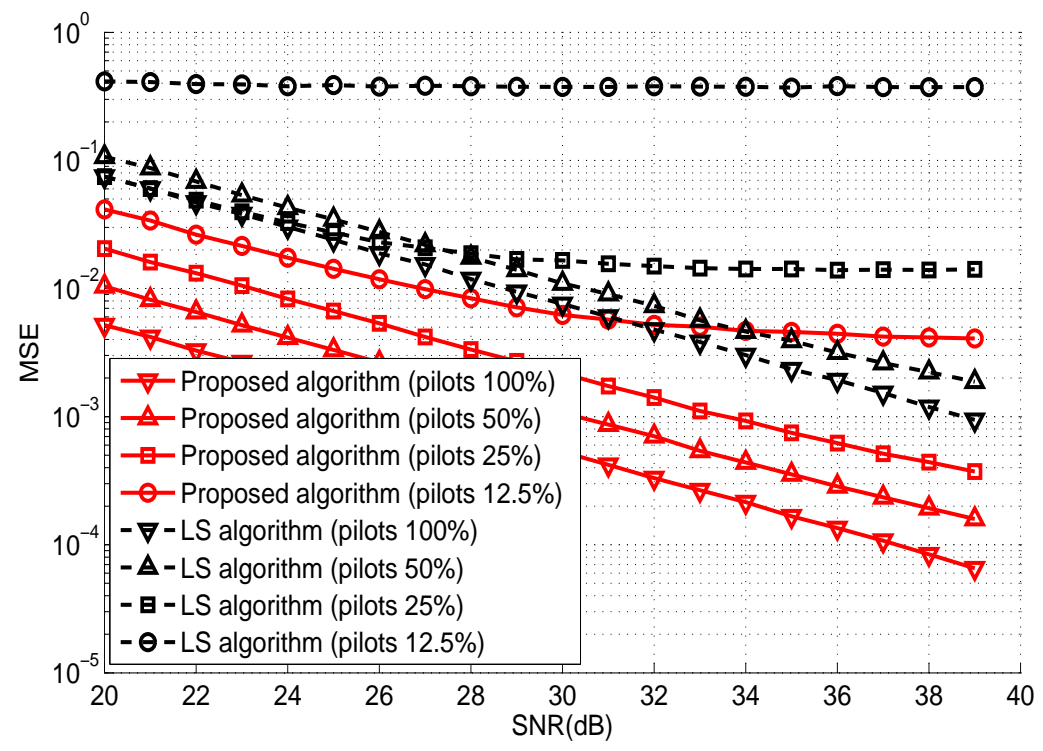

Figure 5: The frequency response MSE comparison of LS-based algorithm and proposed algorithm

Fig.5 shows the MSE (mean square error) performance of frequency response estimation for the LS-based and proposed algorithms with four amounts of pilots being used. For both algorithms, the more pilots are used, the better performance would be. As shown in the figure, all the MSE values decrease with enlarging SNR. If the OFDM-based VLC systems exploit $12.5 \%$ pilots, 
the MSE value of LS-based algorithm is very large even when SNR value is large. While the MSE value of proposed algorithm using $12.5 \%$ pilots is lower than that of LS-based algorithm, and would still gradually drop to a level which is smaller than that of LS-based algorithm using $25 \%$ pilots.

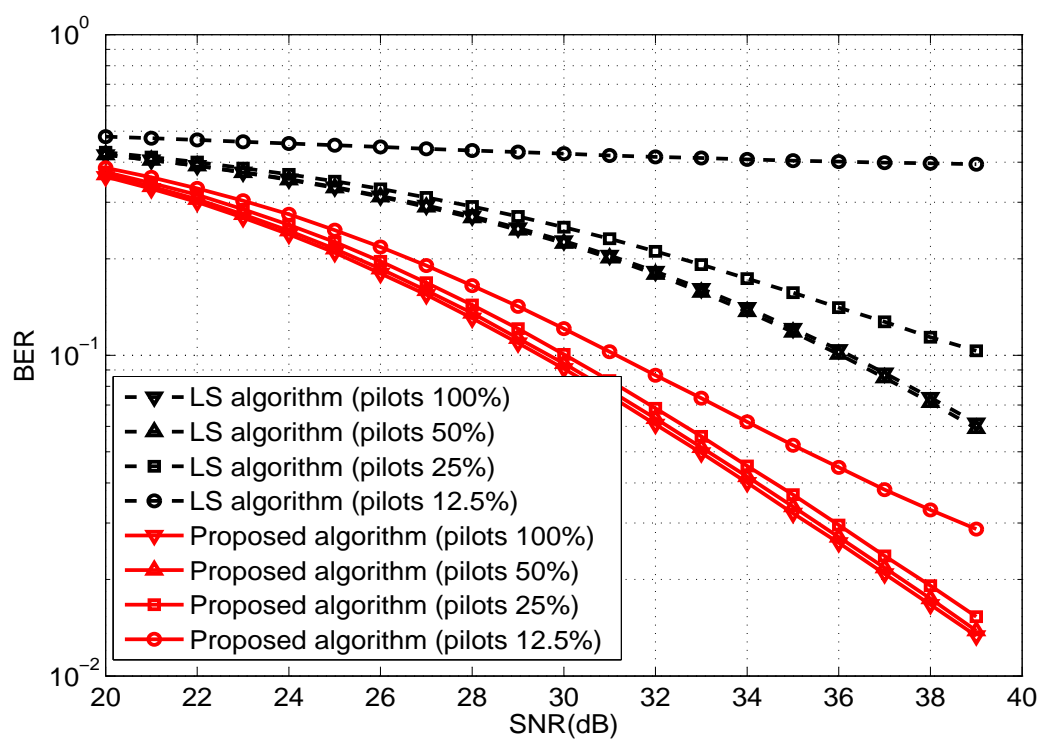

Figure 6: The BER result comparison of LS algorithm and proposed algorithm

Fig.6 shows the BER performance of the LS-based and proposed algorithms. Similar to the situation in frequency response results comparison, when using $12.5 \%$ pilots, the BER performance of LS-based algorithm is almost the same within the $[20 \mathrm{~dB} 40 \mathrm{~dB}]$ SNR range. The BER performance of systems using $100 \%$ pilots is approximately equal to that of $50 \%$ pilots for both algorithms. The proposed algorithm achieves a better BER performance than LS-based algorithm no matter how many pilots are used.

\section{CONCLUSION}

In summary, we have proposed a practical parametric channel estimation algorithm for DCO-OFDM-based VLC systems. When the modulation bandwidth is wide, multipath channels may be non-sparse for the DCO-OFDMbased VLC systems and deteriorate communication performance heavily. Compressive sensing-based channel estimation algorithm cannot be employed 
while our algorithm can work well in this case. Simulation results reveal that our algorithm achieves better performances than the existing LS-based algorithm in both BER and frequency response estimation while does not need complex calculations.

\section{Acknowledgment}

This work was supported by the Mittal Innovation Project of Central South University [Project No. 15MX18] and Postgraduate Innovation Project of Central South University [Project No. 2016zzts228]. This work was also supported by Research Foundation of Education Bureau of Hunan Province.

[1] W. F. Lin, C. W. Chow, C. H. Yeh, Using specific and adaptive arrangement of grid-type pilot in channel estimation for white-lightled-based ofdm visible light communication system, Optics Communications 338 (2015) 7-10.

[2] H. Deng, Peak-to-average power ratio reduction in orthogonal frequency division multiplexing visible light communications system using a combination of a genetic algorithm and a hill-climbing algorithm, Optical Engineering 54 (3) (2015) 036106.

[3] H. Deng, Carrier allocation combined with independent component analysis for multiple-input-multiple-output visible light communication, Optical Engineering 53 (2) (2014) 1-5.

[4] F. Jiang, H. Deng, W. Xiao, S. Tao, K. Zhu, An ica based mimo-ofdm vlc scheme, Optics Communications 347 (2015) 37-43.

[5] S. J. Lee, J. K. Kwon, S. Y. Jung, Evaluation of visible light communication channel delay profiles for automotive applications, EURASIP Journal on Wireless Communications and Networking 2012 (1) (2012) $1-8$.

[6] X. Qian, Y. Deng, H. Deng, Y. Hu, C. Zhang, J. Du, Synchronisation algorithm based on zero correlation code pair for ofdm-based vlc systems, Iet Communications 11. 
[7] J. Du, H. Deng, X. Qian, C. Zhang, Channel estimation scheme based on compressed sensing and parameter estimation for an orthogonal frequency division multiplexing visible light communications system, Optical Engineering 55 (11) (2016) 116109.

[8] Y. S. Hussein, M. Y. Alias, A. A. Abdulkafi, On performance analysis of ls and mmse for channel estimation in vlc systems, in: IEEE International Colloquium on Signal Processing \& ITS Applications, 2016, pp. 204-209.

[9] M. N. I. Khan, M. J. Alam, Noise reduction algorithm for ls channel estimation in ofdm system, in: International Conference on Computer and Information Technology, 2012, pp. 310-315.

[10] W. U. Bajwa, J. Haupt, G. Raz, R. Nowak, Compressed channel sensing, in: Information Sciences and Systems, 2008. Ciss 2008. Conference on, 2008, pp. 5-10.

[11] R. Roy, T. Kailath, Esprit-estimation of signal parameters via rotational invariance techniques, IEEE Transactions on Acoustics Speech \& Signal Processing 37 (7) (1990) 984-995.

[12] T. T. Do, L. Gan, N. Nguyen, T. D. Tran, SPARSITY ADAPTIVE MATCHING PURSUIT ALGORITHM FOR PRACTICAL COMPRESSED SENSING Department of Electrical and Computer Engineering The Johns Hopkins University School of Engineering and Design (2008) 581-587.

[13] X. Zhu, J. Wang, L. Dai, Z. Wang, Sparsity-aware adaptive channel estimation based on snr detection, IEEE Transactions on Broadcasting 61 (1) (2015) 119-126.

[14] O. Narmanlioglu, R. C. Kizilirmak, M. Uysal, Relay-assisted OFDM-based visible light communications over multipath channels, Int. Conf. Transparent Opt. Networks 2015-Augus (2015) 2-5. doi:10.1109/ICTON.2015.7193338.

[15] Y. C. Chi, D. H. Hsieh, C. T. Tsai, H. Y. Chen, H. C. Kuo, G. R. Lin, 450-nm GaN laser diode enables high-speed visible light communication with 9-Gbps QAM-OFDM, Opt Express 23 (10) (2015) 13051-13059. 
doi:10.1364/OE.23.013051.

URL http://www.ncbi.nlm.nih.gov/pubmed/26074558

[16] T. Zhang, S. Guo, H. Chen, F. Zhong, Enhancing the bit error rate of indoor visible light communication systems using adaptive channel estimation algorithm, Iet Communications 9 (4) (2015) 501-507.

[17] U. H. Rohrs, L. P. Linde, Some unique properties and applications of perfect squares minimum phase cazac sequences, in: Communications and Signal Processing, 1992. COMSIG '92., Proceedings of the 1992 South African Symposium on, 1992, pp. 155-160.

[18] T. Report, Lifi channel models office home manufacturing cell (November).

[19] M. Bellanger, FBMC physical layer: a primer, PHYDYAS, January (2010) 1-31.

URL http://www . ict-phydyas .org/teamspace/internal-folder/FBMC-Primer_06-201

[20] M. Uysal, F. Miramirkhani, T. Baykas, N. Serafimovski, V. Jungnickel, Lifi channel models office home manufacturing cell. 